\title{
Accesibilidad en el proceso de admisión a la Universidad de Costa Rica, de la población estudiantil con necesidades educativas especiales asociadas o no a discapacidad
}

\section{Access to University of Costa Rica's admition process for the student with educational needs related or not to disability}

Laura Stiller González CASED

Universidad de Costa Rica San José, Costa Rica laura.stiller@ucr.ac.cr

Martha Gross Martínez Escuela de Orientación y Educación Especial Universidad de Costa Rica

San José, Costa Rica martha.gross@ucr.ac.cr

Resumen: En el presente artículo se identifican aspectos conceptuales y pragmáticos del acceso al proceso de admisión a la Universidad de Costa Rica. Se rescata el marco legal y las acciones que fortalecen la inclusión a la educación superior. Se visualiza el papel de la Universidad de Costa Rica, en sus esfuerzos por hacer efectiva una universidad inclusiva, con alternativas y propuestas acordes a los requerimientos de la población estudiantil con necesidades educativas especiales asociadas o no a discapacidad. Se realiza además, una vinculación entre el Informe del Estado de la Nación 2010 y los aportes de la Universidad de Costa Rica en la construcción de una universidad accesible.

Palabras clave: Accesibilidad, proceso de admisión, educación superior, inclusión educativa, estudiantes con necesidades educativas especiales asociadas o no a discapacidad

\section{Introducción}

El reconocimiento a la diversidad educativa posibilita, entre otros aspectos, que la población estudiantil sea valorada, aceptada y tenga posibilidades de participar en el quehacer universitario.

Desde esta perspectiva, el Estado de la Nación (2010) afirma que en la educación superior como en todos los niveles la equidad es un imperativo; de ahí que se deba procurar responder a una variedad de necesidades y requerimientos de la población estudiantil en general, fundamentada en los principios de diversidad y equidad, proporcionando las oportunidades educativas y de formación, así como 


\begin{abstract}
The following article identifies conceptual and pragmatic aspects of the access in the admission process of the University of Costa Rica. Legal framework and actions that fortify inclusion to superior education are taken into account. The role that the University of Costa Rica plays in the effort to create an inclusive university, with alternatives and proposals that agree with the needs of the students with special educational needs related or not to a disability, is acknowledged. A connection is made between the Report of the State of the Nation 2010 and the contributions of the University of Costa Rica in the construction of an accessible university.
\end{abstract}

Keywords: Accessibility, admition process, superior education, inclusive education, students with special educational needs related or not to a disability los apoyos necesarios para su desarrollo académico.

El presente artículo tiene como propósito dar a conocer y evidenciar los logros alcanzados en materia de admisión a la Universidad de Costa Rica y de la población estudiantil con necesidades educativas especiales asociadas o no a discapacidad. Así pues, esto conlleva a la visualización de una serie de acciones que legitiman el derecho a la formación de los futuros y las futuras profesionales. Además, es importante que los esfuerzos que realiza la Universidad de Costa Rica en la construcción de una universidad accesible, se presenten dentro del Informe del Estado de la Nación.

En el marco de los Principios y Políticas Institucionales en el campo de la discapacidad (Eje 4 Cobertura y Equidad, Accesibilidad) propone desarrollar estrategias para garantizar oportunidades y accesibilidad de todos sus servicios a los grupos con necesidades educativas especiales en todas las sedes universitarias. (Consejo Universitario, 2008)

La Universidad de Costa Rica, como universidad estatal ha ido asumiendo con gran sentido de responsabilidad el principio de igualdad de oportunidades en el proceso de admisión; desde una perspectiva institucional que considera de forma particular y efectiva a las distintas necesidades de la población estudiantil, lo que implica cambios organizativos, metodológicos, así como una demanda de recursos materiales, personales y de formación, según los ajustes razonables correspondientes.

\section{Población estudiantil con necesidades educativas especiales, asociadas o no a discapacidad, en la Universidad de Costa Rica}

La población estudiantil con necesidades educativas especiales, asociadas o no a una discapacidad, es aquella que presenta 
dificultades para acceder a los procesos de enseñanza y aprendizaje, se incluye a personas que tienen deficiencias físicas, mentales, intelectuales o sensoriales.

Esta población que ingresa a la educación superior, va en continuo crecimiento, como resultado de las políticas y legislación inclusivas que se dan desde hace más de una década y que validan el derecho a la educación. En este sentido, Stupp (2005) rescata la importancia de que las universidades hayan ofrecido servicios y apoyos a estudiantes con discapacidad antes de la promulgación de la Ley 7600 de Igualdad de Oportunidades para las personas con discapacidad (1996).

El papel de liderazgo de la Universidad de Costa Rica en este proceso ha representado retos que requirieron una gran dosis de esfuerzo, para romper barreras en la comunidad universitaria en busca de la accesibilidad, la cual se entiende de la siguiente manera:

Accesibilidad significa que las diferentes esferas de participación social que conforman el entorno se encuentren a disposición de todas las personas. Por entorno se debe de entender todas aquellas esferas en las que se desenvuelve el individuo, entre las cuales resaltan el espacio físico, los servicios, las actividades, la información, la documentación y otras. (Lépiz, 1999)

Así, a lo interno de la Universidad de Costa Rica, se han hecho esfuerzos significativos para fortalecer los servicios y estrategias de apoyo, que dan el asesoramiento para lograr una universidad inclusiva. Por lo tanto, las alternativas y propuestas se realizan acordes a las necesidades de dicha población. Entre las instancias que participan están: el Centro de Asesoría y Servicios al Estudiante con Discapacidad (CASED), la Escuela de Orientación y Educación Especial, el Sistema Integrado de Bibliotecas, Documentación e Información (SIBDI), la Comisión Institucional en Materia de Discapacidad (CIMAD), la Comisión Interuniversitaria de acceso a la Educación Superior (CIAES) del Consejo
Nacional de Rectores y el Departamento de Docencia Universitaria (DEDUN), han hecho esfuerzos significativos, por fortalecer los servicios y estrategias de apoyo, que den el asesoramiento y soporte para hacer efectiva una universidad inclusiva, con alternativas y propuestas acordes a las necesidades de dicha población.

\section{Marco legal en materia de accesibilidad}

La igualdad de oportunidades de acceso a la educación superior para estudiantes con necesidades educativas especiales asociadas o no a discapacidad, se sustentan en la Constitución Política de la República en el derecho a la Educación, en la Ley 7600 de Igualdad de Oportunidades para las personas con discapacidad (1996) y la Ley 8661 Convención sobre los Derechos de las personas con discapacidad (2008).

Además, se respalda en la reglamentación particular de las instituciones de educación superior, como el Estatuto Orgánico de la Universidad de Costa Rica (1974), las políticas institucionales en el campo de la discapacidad aprobadas por el Consejo Universitario de la Universidad de Costa Rica (2010-2014) y el Reglamento de Régimen Académico Estudiantil (2011).

La Universidad de Costa Rica, ha definido políticas institucionales que responden a la temática en materia de equidad y accesibilidad, las cuales favorecen la equiparación de oportunidades que permiten la participación de la población universitaria en igualdad de condiciones. Una muestra de ello, son los Principios y Políticas Institucionales de 2009, en donde se anota de forma contundente que la Universidad impulsará acciones dirigidas a reducir y eliminar cualquier tipo de desigualdad en la comunidad universitaria, con el fin de que contribuyan a consolidar una cultura de justicia, equidad y de respeto a las personas (Consejo Universitario, 2008). 
Algunas de estas acciones las realiza el CASED, en coordinación con la Escuela de Orientación y Educación Especial, quienes trabajan en conjunto desde el año 1995, para dar respuestas concretas a la inclusión de estudiantes con discapacidad en las aulas universitarias. Se busca concientizar a la comunidad universitaria sobre el papel de la institución en relación con la temática y ofrecer los servicios y apoyos a la población estudiantil con necesidades educativas especiales, asociada o no a una discapacidad, que les garantice la igualdad de oportunidades en el entorno universitario, desde el momento en que hace la solicitud de ingreso.

Una de las gestiones en este aspecto, ha sido el esfuerzo sistemático y activo en la coordinación entre las instancias involucradas en el proceso de admisión a la Universidad de Costa Rica, (Vicerrectoría de Vida Estudiantil, Instituto de Investigaciones Psicológicas IIP y Escuela de Orientación y Educación Especial), cada uno desde su competencia y experticia, con el fin de que el respeto a la diversidad esté inmerso en la dinámica universitaria.

\section{Admisión y Accesibilidad en la Universidad de Costa Rica}

La Universidad de Costa Rica ha sido una institución de educación superior pionera en el reto de la accesibilidad, a lo largo de los años ha logrado sensibilizar, concienciar a la comunidad universitaria y transmitir que la diferencia de cada persona, es lo que hace que se trabaje por el respeto a ellas, con lo cual se pretende lograr un sistema social y educativo más justo para toda la población universitaria, de esta manera Boff y Muraro (2002) indican que dentro de la igualdad se instaura la diferencia, entendida como apertura del uno al otro, es decir, como reciprocidad.

Los esfuerzos que la Universidad de Costa Rica ha hecho para dar respuesta a los nuevos paradigmas de apoyo y servicios a la población con discapacidad, se sustentan desde una perspectiva de derechos humanos, al fortalecer no sólo la propuesta interna, sino también al brindar asesoría tanto en el ámbito nacional como regional.

Cabra de Luna (2006), plantea que en el nuevo modelo social de la discapacidad, la responsabilidad se traslada del individuo (que se tenía que integrar y adaptar) a la sociedad que debe contar con un diseño accesible para todos y todas. De esta manera, la Universidad de Costa Rica asume el reto de dar respuesta a las necesidades de la población estudiantil con relación al proceso de admisión; al priorizar los requerimientos del estudiante en la ejecución de la prueba de aptitud académica, con el fin de que se desempeñe en igualdad de condiciones, fortaleciendo los componentes $\mathrm{y}$ acciones que le permitan al estudiante contar con una propuesta accesible para su ingreso a la universidad.

El Estado de la Nación plantea en el capítulo 4: La Evolución de la Educación Superior, que la matrícula en las cuatro universidades públicas creció en un $36 \%$; enfatizando que el ingreso a una institución de educación superior depende de una serie de factores: concluir la educación secundaria, la situación socioeconómica del hogar y las becas a las que tengan acceso los y las estudiantes (Estado de la Nación, 2010). Sin embargo, en este importante documento, no se brindan datos de la accesibilidad en el proceso de admisión a la educación superior estatal, ni los esfuerzos conjuntos que se realizan desde el Consejo Nacional de Rectores (CONARE) para otorgar las condiciones requeridas por la población estudiantil con necesidades educativas especiales asociadas o no a discapacidad.

El Informe del Estado de la Nación del 2010 en su introducción señala que: desde su primera entrega ha tenido entre sus propósitos dar un seguimiento detallado de las principales tendencias de la educación superior en el país, tanto en el nivel universitario como en el parauniversitario, 
a partir de la información disponible. No obstante, en este Informe no se detallan aspectos de accesibilidad a la educación superior estatal, es por esto que tratamos de evidenciar el papel del accionar de la Universidad de Costa Rica, en cuanto al ingreso de la población estudiantil con necesidades educativas especiales, asociadas o no a discapacidad, y de esta manera no quede supeditado únicamente a una experiencia interna, lo cual dada la importancia del crecimiento de las oportunidades a la educación superior, merece ser reconocido como una respuesta efectiva hacia la equidad en el ámbito de la educación superior universitaria.

En este sentido, Castellana y Sala (2005) mencionan que la creación de aulas inclusivas es un tema que ha estado especialmente tratado y estudiado en el contexto de la educación primaria y secundaria, pero no en el de los estudios universitarios. De esta manera, adquiere relevancia el esfuerzo por investigar y visibilizar la temática en la educación superior, esto implica retos en la sistematización de los logros alcanzados en la toma de decisiones, así como el apoyo, las acciones y los servicios que se han presentado. Además es importante realizar propuestas transformadoras que permitan y dinamicen la accesibilidad en las aulas universitarias.

La inclusión como proceso en la Universidad de Costa Rica, no sólo es parte de la toma de conciencia que se tiene sobre la temática, sino también en las planificaciones de las instancias vinculadas con la vida académica estudiantil, que articulan los objetivos y acciones encaminadas a la práctica del derecho a la educación.

Desde la práctica, adquiere relevancia la organización previa al ingreso, vinculada a las diferentes etapas del quehacer como son:

establecer lineamientos conjuntos de las universidades estatales desde la CIAES.

acceso a la información: considerando aspectos de formato, diseño y disponibilidad. atención y acompañamiento: por parte de especialistas para dar respuesta a las necesidades educativas especiales asociadas o no a discapacidad, de la población estudiantil que solicita admisión.

Estas etapas se plantean como pilares de la accesibilidad, ya que son elementos claves para el acceso al entorno, a la disposición de la información, al material con formato accesible y a la disponibilidad de un sistema de apoyo para cada estudiante solicitante, ajustándose a las demandas de la diversidad de la población estudiantil.

En el CONARE como parte de la división de coordinación del área de vida estudiantil se encuentra el proyecto de articulación de políticas de accesibilidad en la Educación Superior, perteneciente a la CIAES, que destaca:

Del trabajo realizado por la CIAES y las especialistas de los diferentes programas de atención depende que al y a la estudiante se le equiparen sus oportunidades, y que en la aplicación del examen de admisión se le evalúe con equidad, atendiendo la accesibilidad según los requerimientos personales de cada solicitante. (CIAES, 2011)

Entre los objetivos de la CIAES están: establecer un modelo conjunto para la atención a la población con necesidades educativas especiales que solicitan adecuaciones a la aplicación del examen de admisión de cada universidad; brindar servicios y apoyos a los y las estudiantes con necesidades educativas especiales en los procesos de admisión y permanencia en las universidades estatales, ofrecer capacitación sobre las temáticas de accesibilidad y discapacidad para propiciar la equiparación de oportunidades en la Educación Superior (CIAES, 2011).

A continuación se presentan los datos de inscripción a la Prueba de Aptitud Académica para el 2011 de acuerdo con la universidad de inscripción:

Con respecto a dicha información se observa un total de 1422 solicitudes 
Tabla 1

Inscripciones a la Prueba de Aptitud Académica 2011

\begin{tabular}{lcccccccc}
\hline & UCR & $\begin{array}{c}\text { UCR- } \\
\text { UNA }\end{array}$ & UCR-TEC & $\begin{array}{c}\text { UCR- } \\
\text { UNA- } \\
\text { TEC }\end{array}$ & UNA & TEC & $\begin{array}{c}\text { UNA- } \\
\text { TEC }\end{array}$ & TOTAL \\
\hline AD1 & 292 & 348 & 110 & 303 & 51 & 7 & 10 & 1121 \\
AD2 & 85 & 68 & 20 & 12 & 43 & 5 & 4 & 237 \\
AD3 & 53 & 6 & 4 & 1 & 0 & 0 & 0 & 64 \\
TOTAL & 430 & 422 & 134 & 316 & 94 & 12 & 14 & 1422 \\
\hline
\end{tabular}

Datos de CIAES 2011

de inscripción a la Prueba de Aptitud Académica con adecuaciones, de las cuales 1121 son solicitudes de estudiantes colegiales (AD1); 237 de estudiantes egresados de colegio (AD2) y 64 estudiantes universitarios que repiten su prueba de admisión.

Las áreas de discapacidad o condición por la que solicitan adecuaciones en la prueba de aptitud académica se detallan en la siguiente tabla:

Tabla 2

Distribución de la inscripción por áreas de discapacidad o condiciones

\begin{tabular}{lc}
\hline \multicolumn{1}{c}{ Área/Condición } & Totales \\
\hline Aprendizaje & 1043 \\
Déficit Atencional & 144 \\
Motora & 27 \\
Visual & 72 \\
Auditiva & 32 \\
Emocional & 68 \\
Sistémica & 27 \\
Múltiple & 9 \\
Total & 1422 \\
\hline
\end{tabular}

Datos de CIAES 2011
Es importante aclarar que en la prueba de aptitud académica se señala que las adecuaciones que se brindan a los estudiantes con alguna necesidad educativa especial son de acceso; estas no interfieren con la validez y la confiabilidad del constructo por medir. Estas adecuaciones pretenden igualar las condiciones de aplicación, sin generar ventaja ni desventaja para esta población (Herrera, 2008)

En la tabla 3 se detallan algunas adecuaciones, de acuerdo con el área de discapacidad o condición, requeridas en la prueba de aptitud académica.

De esta forma, en el proceso de admisión se considera que es la persona que solicita su ingreso a la universidad, la que determina los requerimientos de accesibilidad para la realización de la prueba de ingreso, sin detrimento del nivel de exigencia y calidad.

Con esta premisa es que la Universidad de Costa Rica, logra la puesta en común de las instancias especializadas que forman parte del proceso de admisión con adecuación, como son el CASED, Oficina de Registro e Información y el IIP. Las cuales articular y coadyuvan esfuerzos en procura de una atención que ofrece información en formato accesible, acompañamiento de especialistas en la diversidad educativa, espacios de guía y seguimiento para una admisión en una plataforma accesible, tanto de recursos humanos como materiales. 
Tabla 3

Adecuaciones de acuerdo a áreas dediscapacidad o condición

\begin{tabular}{|c|c|}
\hline Condición o Áreas de discapacidad & Adecuaciones \\
\hline Aprendizaje y déficit atencional & $\begin{array}{l}\text { - Tiempo adicional } \\
\text { - Tablas de multiplicar } \\
\text { - Uso de calculadora } \\
\text { - Tamaño del grupo } \\
\text { - Ubicación especial en el aula } \\
\text { - Marcar con x en el folleto }\end{array}$ \\
\hline Visual & $\begin{array}{l}\text { - Tipo y tamaño de letra } \\
\text { - Tiempo adicional } \\
\text { - Prueba impresa en braille } \\
\text { - Versión en audio } \\
\text { - Ubicación especial dentro del aula } \\
\text { - Iluminación del aula } \\
\text { - Apoyo de lector } \\
\text { - Apoyo de escribiente } \\
\text { - Uso de calculadora parlante } \\
\text { - Marcar con x en el folleto }\end{array}$ \\
\hline Auditiva & $\begin{array}{l}\text { - Tiempo adicional } \\
\text { - Uso de diccionario } \\
\text { - Intérprete de LESCO } \\
\text { - Intérprete oral } \\
\text { - Hablar de frente } \\
\text { - Ubicación especial en el aula }\end{array}$ \\
\hline Motora & $\begin{array}{l}\text { - Tiempo adicional } \\
\text { - Descansos } \\
\text { - Mobiliario adaptado } \\
\text { - Escribiente } \\
\text { - Aula accesible }\end{array}$ \\
\hline Emocional & $\begin{array}{l}\text { - Tamaño de grupo } \\
\text { - Tiempo adicional } \\
\text { - Ubicación especial en el aula } \\
\text { - Marcar con x en el folleto }\end{array}$ \\
\hline Sistémica & $\begin{array}{l}\text { - Tiempo adicional } \\
\text { - Tamaño de grupo } \\
\text { - Mobiliario adaptado } \\
\text { - Escribiente } \\
\text { - Lector } \\
\text { - Aula accesible } \\
\text { - Marcar con x en el folleto }\end{array}$ \\
\hline Múltiple & $\begin{array}{l}\text { - Tiempo adicional } \\
\text { - Tamaño de grupo } \\
\text { - Mobiliario adaptado } \\
\text { - Escribiente } \\
\text { - Lector } \\
\text { - Aula accesible } \\
\text { - Marcar con x en el folleto }\end{array}$ \\
\hline
\end{tabular}

Fuente: Datos de CIAES 2011 


\section{Conclusiones}

De conformidad con la normativa internacional Ley 8661: Convención sobre los Derechos de la personas con Discapacidad y su protocolo facultativo; la Ley 7600 de Igualdad de Oportunidades para las Personas con Discapacidad; las políticas promulgadas por el Consejo Universitario de la Universidad de Costa Rica; la normativa de admisión a la Universidad de Costa Rica y el Reglamento de Régimen Académico Estudiantil, se ha establecido el encuadre legal que ha sustentado las acciones para el ingreso a la educación superior de los y las estudiantes con necesidades educativas especiales asociadas o no a discapacidad en igualdad de oportunidades.

La Universidad de Costa Rica busca los medios que permitan continuar reforzando e innovando no sólo el marco jurídico de referencia al tema, sino también las estrategias que posibiliten una universidad accesible. Es por consiguiente, que en este artículo se reflejan algunas de las acciones en el proceso de admisión para la población con necesidades educativas especiales asociadas o no a discapacidad, y proponemos que es importante visibilizar en los próximos Informes del Estado de la Nación, estos y otros esfuerzos institucionales que se ejecutan en materia de accesibilidad a la educación superior universitaria.

CIAES realiza coordinaciones entre las universidades estatales, para la inscripción conjunta a la prueba de aptitud académica. Y a nivel intrauniversitario se coordina entre las instancias de la Vicerrectoría de Vida Estudiantil: Oficina de Registro e Información, CASED, y con el IIP para atender a la población con necesidades educativas especiales asociadas o no a una discapacidad; según sus requerimientos en materia de accesibilidad al proceso de admisión. Estos aportes se constituyen en insumos en la construcción de una Universidad accesible para todos y todas.

\section{Referencias bibliográficas}

Asamblea Legislativa de Costa Rica. (1996). Ley 7600: Ley de Igualdad de Oportunidades para las Personas con Discapacidad. San José: Diario Oficial la Gaceta.

Asamblea Legislativa de Costa Rica. (2008). Ley 8661: Convención sobre los Derechos de las personas con Discapacidad y su protocolo facultativo. San José: Diario Oficial la Gaceta.

Boff, L. y Muraro, R.M. (2002). Femenino y masculino. Una nueva conciencia para el encuentro de las diferencias. Madrid: Trotta Ediciones.

Cabra de Luna, M. (2006). Igualdad de Oportunidades, No Discriminación y Accesibilidad Universal de las Personas con Discapacidad. Madrid: Memoria de X Jornadas sobre Derecho $\mathrm{y}$ situaciones de discapacidad.

Castellana, M. y Sala, I. (2005). La universidad ante la diversidad en el aula. Revista Aula abierta, Revista del Instituto de Ciencias de la Educación de la Universidad de Barcelona, (85), 57-83.

CIAES. (2011). Articulación de políticas de accesibilidad en la Educación Superior. Informe de proyecto. Coordinación del área de vida estudiantil de CONARE.

CONARE. (2010). La evolución de la Educación Superior, Costa Rica. En Estado de la Nación.

Consejo Universitario. (2008a). Políticas de la Universidad de Costa Rica para el año 2009. Universidad de Costa Rica.

Consejo Universitario. (2008b). Políticas de la Universidad de Costa Rica para los años 2010 - 2014. Universidad de Costa Rica.

Herrera, E. (2008). La prueba de aptitud académica en el contexto de las necesidades educativas especiales. Informe de trabajo. Instituto 
de Investigaciones Psicológicas, Universidad de Costa Rica.

Lépiz, O. (1999). Derechos Humanos y la accesibilidad del entorno. San José: Memoria de conferencia presentada en la Jornada de reflexión sobre aspectos Conceptuales y Vivencias relacionados con la Igualdad de Oportunidades para las Personas con discapacidad.

Stupp, R. (2005). Integración de Personas con Discapacidad a las Instituciones de Educación Superior en Costa Rica. Informe para UNESCO.

Vicerrectoría de Vida Estudiantil: Unidad de estudio y asesoría. (2011). Las adecuaciones de acceso en la aplicación de la prueba de aptitud académica, para estudiantes con necesidades educativas especiales asociadas a una discapacidad. Informe de trabajo. Universidad de Costa Rica. 